\title{
Estimation par conductimétrie de la flore aérobie mésophile, des bactéries coliformes et de la flore psychrotrophe du lait cru
}

\author{
C Piton, D Rongvaux-Gaïda \\ INRA, Station de recherches en technologie et analyses laitières, BP 89, 39800 Poligny, France
}

(Reçu le 5 février 1990; accepté le 30 mars 1990)

\begin{abstract}
Résumé - Cent trente échantillons de lait cru contenant entre $10^{4}$ et $6,6 \cdot 10^{7} \mathrm{UFC} / \mathrm{ml}$ ont été analysés en double, par conductimétrie par le Microbiological Growth Analyser AT Malthus, selon les conditions expérimentales suivantes : en cellules de $2 \mathrm{ml}$ sur Spye Broth à $30^{\circ} \mathrm{C}\left(\mathrm{TD} 30^{\circ} \mathrm{C}\right.$ ), sur Spye Broth à $10^{\circ} \mathrm{C}\left(\operatorname{TD} 10^{\circ} \mathrm{C}\right.$ ), sur Spye Broth additionné de $2 \mathrm{mg} / 1$ de cristal violet à $30^{\circ} \mathrm{C}$ (TD CV) et en cellules de $10 \mathrm{ml}$ sur Coliform Broth à $30^{\circ} \mathrm{C}$ (TD Coli). Ils ont également été soumis à la numération de la flore totale après agitation standard (FTAS) et après agitation pendant $30 \mathrm{~s}$ à l'aide d'un Ultra-turrax tournant à $20000 \mathrm{t} / \mathrm{min}$ (FTUT); les bactéries coliformes et les micro-organismes psychrotrophes ont été dénombrés uniquement après agitation par Ultra-turrax. Tous les ensemencements ont été effectués à l'aide de l'ensemenceur Spiral. Avec un inoculum de $0,1 \mathrm{ml}$ de lait dans les cellules, le coefficient de corrélation entre le logarithme du nombre d'UFC/ml (FTAS) et le temps de détection TD $30^{\circ} \mathrm{C}$ est de $-0,822$ et l'écart type résiduel de la régression Référence sur TD $30^{\circ} \mathrm{C}$ de $0,386 \log (\mathrm{UFC} / \mathrm{ml})$. Cette précision se dégrade de façon significative avec un inoculum de $1 \mathrm{ml}$ de lait $\left[s_{y, x}=0,452 \log (\mathrm{UFC} / \mathrm{ml})\right]$ ou si les numérations ont été effectuées après une agitation par Ultra-turrax $\left[s_{y, x}=0,467 \mathrm{log}(\mathrm{UFC} / \mathrm{ml})\right]$. Le coefficient de corrélation entre le logarithme du nombre de coliformes et le temps de détection sur Coliform Broth TD Coli est de - 0,823 et l'écart type résiduel de la régression Référence sur TD Coli de 0,424 log (UFC/ml). Pour la flore psychrotrophe, ces paramètres sont respectivement de $-0,667$ et de $0,640 \mathrm{log}$ (UFC/ml) pour la régression entre le nombre d'UFC/ml (en log) et le temps de détection TD CV et respectivement de - 0,945 et de 0,287 $\log (\mathrm{UFC} / \mathrm{ml})$ pour la régression entre le nombre d'UFC/ml (en log) et le temps de détection TD 10 ${ }^{\circ} \mathrm{C}$. Quel que soit le groupe microbien, l'écart type relatif de répétabilité des temps de détection (en h) est faible $(2-4 \%)$ et constant à tous les niveaux de contamination du lait. Par contre, après conversion en UFC/ml à partir de la pente de la droite de calibrage, l'écart type de répétabilité augmente quand le niveau de contamination du lait baisse. Pour la flore totale, l'écart type relatif géométrique de répétabilité est en moyenne de $35,9 \%$ et dépasse $40 \%$ au-dessous de $10^{5} \mathrm{UFC} / \mathrm{ml}$.
\end{abstract}

conductimétrie / Malthus / lait cru / flore totale / flore psychrotrophe / coliforme / justesse / répétabilité

Summary - Estimation of total aerobic mesophilic flora, coliform bacteria and psychrotrophic flora in raw milk by conductimetry. A series of 130 raw milk samples ranging from $10^{4}$ to $6.6 \times 10^{7} \mathrm{CFU} / \mathrm{ml}$ were analysed in duplicate by conductimetry with the Microbiological Growth Analyser AT Malthus, using the following experimental conditions: in $2 \mathrm{ml}$ cells on Spye Broth at $30^{\circ} \mathrm{C}$ $\left(D T 30^{\circ} \mathrm{C}\right)$, on Spye Broth at $10^{\circ} \mathrm{C}\left(D T 10^{\circ} \mathrm{C}\right)$, on Spye Broth supplemented with crystal violet (2 $\mathrm{mg} / \mathrm{l})$ at $30^{\circ} \mathrm{C}$ (DT CV) and in $10 \mathrm{ml}$ cells on Coliform Broth at $30^{\circ} \mathrm{C}$ (TD Coli). Using the Spiral Plate Maker, they have also been submitted to the enumeration of total flora after a standard shaking (TFSS) or after shaking with an Ultra-turrax for $30 \mathrm{~s}$ at $20000 \mathrm{rpm}$ (TFUT); coliform bacteria and psychrotrophic flora have been enumerated only after shaking with an Ultra-turrax. With an $0.1 \mathrm{ml}$ inoculation volume in cells, the coefficient of correlation between the logarithms of the numbers of 
CFU/mI (TFSS) and the detection times DT $30^{\circ} \mathrm{C}$ is -0.822 and the residual standard deviation of the regression line Reference on TD $30^{\circ} \mathrm{C}$ is $0.386 \log (\mathrm{CFU} / \mathrm{ml})$. This accuracy decreases with an inoculation volume of $1 \mathrm{ml}$ in cells $\left[s_{y, x}=0.452 \mathrm{log}(C F U / \mathrm{ml})\right]$ or with the shaking of samples with an Ultra-turrax before plating $\left[s_{y, x}=0.467 \log (C F U / m /)\right]$. The coefficient of correlation between the numbers of coliforms (in log) and the detection times TD Coli is - 0.823 and the residual standard deviation of the regression line Reference on DT Coli is $0.424 \mathrm{log}(C F U / \mathrm{ml})$. For the psychrotrophic flora, these parameters are respectively $(-0.667$ and $0.640 \mathrm{log} C F U / \mathrm{ml})$ for the regression between the numbers of CFU/ml (in log) and the detection times DT CV, and respectively -0.945 and $0.287 \mathrm{log}$ (CFU/mI) for the regression between the numbers of CFU/mI (in log) and the detection times $D T 10^{\circ} \mathrm{C}$. Whatever the microbial group, the repeatability relative standard deviation of the detection times (in $h$ ) averages 2 to $4 \%$ and is constant at the various levels of milk contamination. Expressed in CFU/m/ from the slope of the regression line, the repeatability standard deviation becomes proportionally higher as the level of milk contamination decreases. For total flora, the geometric relative standard deviation of repeatability averages $35.9 \%$ and is over $40 \%$ for the low levels of contamination (<10 $\mathrm{CFU} / \mathrm{ml})$.

conductimetry / Malthus / raw milk / total flora / psychrotrophic flora / coliform / accuracy / repeatability

\section{INTRODUCTION}

Dans un milieu nutritif peu tamponné, la croissance et le métabolisme microbiens se traduisent par des changements significatifs de conductance (Firstenberg-Eden et Zindulis, 1984). Ces changements sont liés à la dégradation de ses substrats en molécules plus petites, plus mobiles et d'une charge électrique plus élevée comme par exemple, l'hydrolyse des protéines en acides aminés ou celle des hydrates de carbone en lactate. Généralement, cette production d'ions devient suffisante pour entraîner une modification significative de la conductance du milieu (seuil de détection) lorsque le niveau de la population microbienne dans la cuvette de mesure a atteint $10^{6}-10^{8}$ microorganismes/ml (Hadley et Senyk, 1975; Firstenberg-Eden et Eden, 1984; Suhren et Heeschen, 1985). Le temps nécessaire pour atteindre ce seuil de détection est d'autant plus grand que le niveau initial de contamination de l'échantillon est faible.

L'utilisation des mesures de conductance pour l'appréciation de la qualité bactériologique du lait cru a déjà fait l'objet de nombreux travaux (Cady et al, 1978; O'Connor, 1979; Gnan et Luedecke, 1982; Firstenberg-Eden et Tricarico, 1983; Firstenberg-Eden, 1984; Suhren et Heeschen, 1985; Nieuwenhof et Hoolwerf, 1987; Piton et Dasen, 1988). D'autres auteurs se sont intéressés à cette technique pour l'estimation de groupes microbiens déterminés : les bactéries coliformes (Firstenberg-Eden et al, 1984) ou la flore psychrotrophe (Firstenberg-Eden et Tricarico, 1983; Coombs, 1989). Mais les conditions expérimentales (milieu de culture et/ou température d'incubation; type de matériel) varient d'une étude à l'autre, de même que les paramètres statistiques d'évaluation (tableau I). D'autre part, la quasi-totalité des travaux publiés sur les mesures de conductance ont été effectués sur les différentes versions de l'appareil commercialisé par Bactomatic.

Le premier objectif de notre étude a donc été d'établir, pour l'estimation de la flore totale du lait cru, toutes les caractéristiques analytiques des mesures de conductance sur le Microbiological Growth Analyser commercialisé par la société Malthus. En effet, les caractéristiques de l'ap- 
Tableau I. Justesse des mesures de conductance pour l'estimation de la qualité bactériologique du lait cru (Données bibliographiques).

Accuracy of conductimetry for the evaluation of the bacteriological quality of raw milk.

\begin{tabular}{|c|c|c|c|c|c|c|c|}
\hline \multirow[t]{2}{*}{ Référence } & \multicolumn{6}{|c|}{ Régression } & \multirow[t]{2}{*}{ Remarques } \\
\hline & $\mathrm{n}$ & $\begin{array}{l}\text { Étendue } \\
\log (U F C / m I)\end{array}$ & 1) & a & $s_{y, x}$ & $r$ & \\
\hline Cady et al (1978) & 27 & $2-6$ & & & & $-0,80$ & Bactometer 32 \\
\hline O'Connor (1979) & $\begin{array}{l}230 \\
93\end{array}$ & $\begin{array}{l}3-7 \\
3-7\end{array}$ & $\begin{array}{l}-0,242 \\
-0,311\end{array}$ & $\begin{array}{l}7,038 \\
7,853\end{array}$ & $\begin{array}{l}0,64 \\
0,54\end{array}$ & $\begin{array}{l}-0,67 \\
-0,69\end{array}$ & Bactometer 32 \\
\hline Gnan et Luedecke (1982) & 151 & $3-7$ & & & & $-0,88$ & Bactometer 32 \\
\hline $\begin{array}{l}\text { Firstenberg-Eden } \\
\text { et Tricarico (1983) }\end{array}$ & 110 & $3-8$ & $-0,24$ & 9,07 & & $-0,96$ & $\begin{array}{l}\text { Bactometer } 120 \\
\text { On-MPCA }-18^{\circ} \mathrm{C}\end{array}$ \\
\hline \multirow[t]{2}{*}{$\begin{array}{l}\text { Nieuwenhof et Hoolwerf } \\
\text { (1987) }\end{array}$} & 108 & $4-6$ & $-0,29$ & 7,65 & 0,40 & & $\begin{array}{l}\text { Bactometer } 123 \\
\text { On-MPCA }-30^{\circ} \mathrm{C}\end{array}$ \\
\hline & 119 & $4-6$ & $-0,21$ & 6,70 & 0,33 & & $\begin{array}{l}\text { Bactometer } 123 \\
\text { In-MPCA }-30^{\circ} \mathrm{C}\end{array}$ \\
\hline \multirow[t]{3}{*}{ Piton et Dasen (1988) } & 128 & $3-8$ & $-0,092$ & 7,306 & 0,591 & $-0,812$ & $\begin{array}{l}\text { Bactometer } 123 \\
\text { On-MPCA }-18^{\circ} \mathrm{C}\end{array}$ \\
\hline & 134 & $4-7$ & $-0,200$ & 6,777 & 0,465 & $-0,676$ & $\begin{array}{l}\text { Bactometer } 123 \\
\text { On-MPCA }-30^{\circ} \mathrm{C}\end{array}$ \\
\hline & 134 & $4-7$ & $-0,177$ & 6,345 & 0,492 & $-0,626$ & $\begin{array}{l}\text { Bactometer } 123 \\
\text { In-MPCA }-30^{\circ} \mathrm{C}\end{array}$ \\
\hline
\end{tabular}

$n$ : Nombre d'échantillons, number of samples; $b$ : pente de la droite de calibrage, slope of the calibration line; $a$ : ordonnée à l'origine de la droite de calibrage, intercept of the calibration line; $s_{y, x}$ : écart type résiduel de la régression en $\log (\mathrm{UFC} / \mathrm{ml})$, residual standard deviation of the regression in $\log (\mathrm{CFU} / \mathrm{ml}) ; r$ : coefficient de corrélation, correlation coefficient.

pareillage (type d'électrodes en particulier) et les conditions opératoires (nature et composition du milieu) pouvaient conduire à des paramètres analytiques différents de ceux obtenus récemment par Nieuwenhof et Hoolwerf (1987) ou Piton et Dasen (1988) sur le Bactometer 123. Dans une deuxième phase, nous avons également testé l'utilisation de cet appareil pour l'estimation du nombre de bactéries coliformes et de micro-organismes psychrotrophes, comme l'on disposait de peu de critères objectifs sur ces applications. Dans les travaux précédents sur l'estimation de la flore psychrotrophe par conductimétrie, les auteurs ont choisi une température d'incubation légèrement plus faible que celle utilisée pour la flore totale, de façon à limiter la croissance des micro-organismes mésophiles : $18^{\circ} \mathrm{C}$ après une préincubation des échantillons de $7 \mathrm{~h}$ à $10^{\circ} \mathrm{C}$ (FirstenbergEden et Tricarico, 1983) ou $20{ }^{\circ} \mathrm{C}$ (Coombs, 1989). Dans notre essai, nous avons testé 2 nouvelles voies : d'une part, 
l'utilisation, à $30{ }^{\circ} \mathrm{C}$, d'un inhibiteur de croissance de la flore à Gram positif puisque la majorité des micro-organismes psychrotrophes sont à Gram négatif; d'autre part, une température d'incubation de $10^{\circ} \mathrm{C}$ de façon à limiter au maximum la croissance de la flore mésophile.

\section{MATÉRIEL ET MÉTHODES}

\section{Origine des échantillons}

Cent trente échantillons de lait cru ont été prélevés et conservés à $0^{\circ} \mathrm{C}$, au plus $24 \mathrm{~h}$ après leur prélèvement. Tous provenaient de citernes de ramassage de 4 grandes entreprises laitières.

\section{Numérations microbiennes}

Les échantillons de lait cru ont été répartis, après homogénéisation, en 2 fractions de $25 \mathrm{ml}$ dans des flacons de $60 \mathrm{ml}$ : l'une d'elles a subi une agitation standard consistant en 25 mouvements aller-retour d'une amplitude de $25-30 \mathrm{~cm}$ effectués en 7-10 s (Anonyme, 1978); l'autre fraction a subi une agitation pendant $30 \mathrm{~s}$ à l'aide d'un homogénéisateur à turbine (Ultraturrax, type 18/10) tournant à 20000 tours/min. Ce dernier mode d'agitation est actuellement celui qui assure la meilleure dispersion des amas microbiens (Richard, 1980; Richard et al, 1981).

Les fractions agitées de façon standard et par turbine ont été soumises au dénombrement de la flore aérobie mésophile sur milieu Plate Count Agar (Difco) après $3 \mathrm{j}$ d'incubation à $30^{\circ} \mathrm{C}$. D'autre part, les fractions agitées par turbine ont été soumises au dénombrement des groupes microbiens suivants :

- bactéries coliformes sur milieu Violet Red Bile Agar (Difco) après $24 \mathrm{~h}$ d'incubation à $30{ }^{\circ} \mathrm{C}$;

- flore psychrotrophe sur milieu Plate Count Agar (Difco) après $10 \mathrm{j}$ d'incubation à $7{ }^{\circ} \mathrm{C}$;
- flore à Gram négatif sur milieu Nutrient Agar (Institut Pasteur) additionné de cristal violet à la concentration de $2 \mathrm{mg} /$, après $3 \mathrm{j}$ d'incubation à $30^{\circ} \mathrm{C}$.

Les ensemencements ont été réalisés à la surface de la gélose à l'aide de l'appareil Spiral décrit par Gilchrist et al (1973). Nous avons choisi cette méthode comme technique de référence car il a été largement montré qu'elle donne des résultats similaires à ceux de la technique classique de dénombrement (Campbell et Gilchrist, 1973; Gilchrist et al, 1973; Donnelly et al, 1976; Jarvis et al, 1977; Peeler et al, 1977).

Les analyses ont été effectuées en double pour chaque échantillon, afin d'obtenir une meilleure précision des résultats de numération. Les résultats sont exprimés en unités formant colonies par millilitre de lait (UFC/ml).

\section{Mesures de conductance}

Le Microbiological Growth Analyser AT commercialisé par Malthus est composé de 1 ou 2 incubateurs (entre $+5^{\circ} \mathrm{C}$ et $+45^{\circ} \mathrm{C}$ ) pouvant recevir 128 cellules de mesure, d'un système d'acquisition de données (DAS) et d'un ordinateur. Chaque cellule de mesure est composée d'un tube de verre contenant le bouillon de culture et de 2 électrodes de platine sur une plaque de céramique. La fonction du DAS est de mesurer, à intervalles réguliers (toutes les $6 \mathrm{~min}$ ), la conductance du milieu de culture dans chaque cellule et de stocker provisoirement ces informations avant leur transfert à l'ordinateur. Cet ordinateur assure le stockage et le traitement des informations. En particulier, un algorithme permet de déterminer automatiquement, pour chaque échantillon, le temps de détection, c'est-àdire le temps à partir duquel on peut mesurer une augmentation significative de la conductance dans le milieu.

Les conditions expérimentales sont rassemblées sur le tableau II. Dans chaque cas, le milieu liquide est réparti stérilement dans les cellules de mesure, à raison de $2 \mathrm{ml}$ ou de $9 \mathrm{ml}$ par tube selon le type de cellule. II est ensuite inoculé avec l'échantillon de lait (agité de façon standard) et les cellules sont aussitôt placées dans l'incubateur. 
Tableau II. Conditions expérimentales testées sur l'appareil Malthus. Experimental conditions tested with the Microbiological Growth Analyser Malthus.

\begin{tabular}{lclll}
\hline Groupe microbien & $\begin{array}{c}\text { Cellules de } \\
\text { mesure }\end{array}$ & Milieu de culture* & Température & $\begin{array}{c}\text { Dose } \\
\text { d'inoculum }\end{array}$ \\
\hline $\begin{array}{l}\text { Flore totale } \\
\text { Bactéries coliformes }\end{array}$ & $2 \mathrm{ml}$ & $\begin{array}{l}\text { Spye Broth } \\
\text { Coliform Broth }\end{array}$ & $\begin{array}{l}30^{\circ} \mathrm{C} \\
30^{\circ} \mathrm{C}\end{array}$ & $\begin{array}{l}1 \text { et } 0,1 \mathrm{ml} \\
1 \mathrm{ml}\end{array}$ \\
Flore psychrotrophe & $2 \mathrm{ml}$ & $\begin{array}{l}\text { Spye Broth }+ \\
\text { cristal violet } 2 \mathrm{mg} / \mathrm{l} \\
\text { Spye Broth }\end{array}$ & $30^{\circ} \mathrm{C}$ & 1 et $0,1 \mathrm{ml}$ \\
& $2 \mathrm{ml}$ & $10^{\circ} \mathrm{C}$ & 1 et $0,1 \mathrm{ml}$ \\
\hline
\end{tabular}

* Spye Broth et Coliform Broth commercialisés par Malthus.

\section{Calculs statistiques}

La répétabilité et la justesse des mesures de conductance ont été calculées conformément aux recommandations de la norme FIL 128 (Anonyme, 1985); avant les calculs statistiques, les nombres d'UFC/ml ont subi une transformation logarithmique de base 10 destinée à normaliser la distribution.

\section{Justesse}

La justesse représente l'étroitesse de l'accord entre la valeur vraie de la grandeur à mesurer et le résultat moyen qui serait 'obtenu en appliquant la technique expérimentale un grand nombre de fois (Anonyme, 1985). La justesse d'une technique est évaluée en comparant la valeur vraie avec la moyenne de plusieurs déterminations obtenues avec la technique expérimentale, de façon à réduire les erreurs de fidélité. Par convention, la valeur vraie est donnée par le nombre d'UFC/ml obtenu avec les méthodes classiques de dénombrement (dans notre cas, avec l'appareil Spiral).

Les résultats antérieurs ont déjà clairement établi que, pour les niveaux habituels de contamination du lait cru (moins de $10^{7} \mathrm{UFC} / \mathrm{ml}$ ), il existe une relation linéaire entre le temps de détection et le logarithme du nombre d'UFC/ml (Cady et al, 1978; O'Connor, 1979; Gnan et Luedecke, 1982; Firstenberg-Eden et Tricarico, 1983; Nieuwenhof et Hoolwerf, 1987; Piton et Dasen, 1988). Conformément à la norme FIL, l'équation de calibrage $(y=b x+a)$ a été établie à partir des moyennes des 2 déterminations faites sur chaque échantillon par les 2 techniques; le logarithme décimal du nombre d'UFC/ $\mathrm{ml}$ est considéré comme variable dépendante et le temps de détection comme variable indépendante, pour donner une estimation de l'écart type résiduel dans l'unité de la technique de référence. Nous avons appliqué la norme FIL établie pour les méthodes chimiques, bien que certaines hypothèses ne soient plus vérifiées en microbiologie (en particulier, une erreur négligeable dans la mesure des $x$ et des $y$ ).

La précision d'estimation de la technique expérimentale est donnée par les limites de l'intervalle de confiance à l'intérieur duquel se situe, avec une probabilité de $95 \%$, la vraie valeur donnée par la méthode de référence. Si y est le nombre d'UFC/ml calculé à partir de la droite de calibrage, ces limites sont, en valeur logarithmique, $\log y \pm 1,96 \cdot s_{y, x}$ où $s_{y, x}$ est l'écart type résiduel des écarts à la droite de régression. En données non transformées, ces limites sont $y / 10^{1,96 s_{y, x}}$ pour la limite inférieure et $y \cdot 10^{1,96 s_{y, x}}$ pour la limite supérieure. 


\section{Répétabilité}

La variance de répétabilité des temps de détection $\left(s_{r}^{2}\right)$ a été établie à partir des mesures de conductance effectuées en double sur chaque échantillon, selon la formule suivante :

$$
s_{r}^{2}=\frac{1}{2 n} \sum_{i=1}^{n} w_{i}^{2}
$$

où $w_{i}$ est la valeur absolue de la différence entre doubles pour l'échantillon $i$ et $n$ le nombre d'échantillons analysés (Anonyme, 1985). Dans le cas des temps de détection, l'écart type de répétabilité est alors exprimé en $\mathrm{h}$; mais pour pouvoir comparer valablement cet écart type avec ceux des autres techniques, nous l'avons également exprimé en log (UFC/ml) grâce à la formule :

$$
s_{r}^{*}[\mathrm{en} \log (\mathrm{UFC} / \mathrm{ml})]=b \cdot s_{r}
$$

où $b$ est la pente de la droite de calibrage. Une telle conversion présente un réel intérêt; en effet, 2 techniques qui donnent le même écart type de répétabilité exprimé dans l'unité d'origine, ont en fait des répétabilités exprimées en $\mathrm{UFC} / \mathrm{ml}$ très différentes, si les pentes des équations de calibrage sont différentes.

L'écart type relatif géométrique de répétabilité GRSD en données non transformées peut alors être estimé à partir de la formule suivante :

$$
\text { GRSD (en UFC/ml) }=\left(10^{s^{*}}-1\right) \cdot 100
$$

Ce paramètre exprime la variabilité des résultats au-dessus de la moyenne géométrique (Piton et Grappin, 1990).

\section{RÉSULTATS}

\section{Estimation de la flore totale}

Le tableau III présente la répétabilité et la justesse des mesures de conductance, obtenues à partir d'une population de 130 échantillons de lait cru pour l'estimation de

Tableau III. Répétabilité et justesse des mesures de conductance pour l'estimation de la flore totale du lait cru, en fonction de la dose d'inoculum des échantillons de lait dans les cellules de mesure. Influence of the milk volume of inoculation in the cells on the repeatability and the accuracy of the conductance measures for the estimation of total flora in raw milk.

\begin{tabular}{llllllll}
\hline $\begin{array}{c}\text { Dose } \\
\text { d'inoculum }\end{array}$ & $\begin{array}{c}\text { Temps } \\
\text { moyen }\end{array}$ & $\begin{array}{c}\text { Mode } \\
\text { d'agitation }\end{array}$ & $\mathrm{s}_{\mathrm{r}}{ }^{*}$ & GRSD & Équation de régression & $\mathrm{s}_{\mathrm{y}, \mathrm{x}}$ \\
\hline \multirow{2}{*}{$1 \mathrm{ml}$} & \multirow{2}{*}{$7,86 \mathrm{~h}$} & AS & 0,126 & $38,8 \%$ & $Y=-0,397$ & $X+8,306$ & 0,452 \\
& & UT & 0,129 & $34,7 \%$ & $Y=-0,406$ & $X+8,709$ & 0,539 \\
$0,1 \mathrm{ml}$ & $8,50 \mathrm{~h}$ & AS & 0,133 & $35,9 \%$ & $Y=-0,445$ & $X+8,966$ & 0,386 \\
& & UT & 0,139 & $37,8 \%$ & $Y=-0,465 X+9,471$ & 0,467 \\
\hline
\end{tabular}

Mode d'agitation des échantillons pour la numération de la flore totale : Shaking method of the samples.

AS : agitation de façon standard, standard shaking; UT : agitation par turbine (Ultra-turrax), shaking with an Ultraturrax for $30 \mathrm{~s}$ at $20000 \mathrm{rm} ; \mathrm{s}_{\mathrm{r}}{ }^{*}$ : écart type de répétabilité des temps de détection (en log UFC/ml, calculé à partir de la pente de la droite de calibrage, repeatability standard deviation of the detection times in log (CFU/ml); GRSD: écart type relatif géométrique de répétabilité (en UFC/ml), geometric relative standard deviation of repeatability in $\log$ (in CFU/ml); Y : logarithme du nombre d'UFC/ml obtenu après agitation standard ou turbine, logarithm of the number of CFU/ml; X : temps de détection (en h) après agitation standard des échantillons, detection time (h); $s_{y, x}$ : écart type résiduel de la régression en log (UFC/ml), residual standard deviation of the regression in log (CFU/ml); $*$ : temps moyen de détection (moyenne des 128 à 130 déterminations en double), mean detection time (mean of the 128 or 130 determinations in duplicate). 
la flore totale. On observe tout d'abord que, quelle que soit la dose d'inoculum, la précision d'estimation de ces mesures est meilleure lorsque les numérations microbiennes ont été réalisées après une agitation standard : par exemple, pour un inoculum de $0,1 \mathrm{ml}$, celle-ci passe de $\pm 1,96 \times$ 0,386 , soit $\pm 0,757 \log (\mathrm{UFC} / \mathrm{ml})$ dans le cas d'une agitation standard, à $\pm 0,915$ log (UFC/ml) dans le cas d'une agitation par turbine. La différence est d'ailleurs significative au seuil de $5 \%$, pour les 2 doses d'inoculum testées (test $F$ de comparaison des variances à $n-2$ dl; Dagnelie, 1970). D'autre part, la précision d'estimation est significativement plus faible au seuil de $5 \%$ pour un inoculum de $0,1 \mathrm{ml}$ de lait que pour un inoculum de $1 \mathrm{ml}$.

La figure 1 présente la relation obtenue entre les temps de détection à $30^{\circ} \mathrm{C}$ avec
$0,1 \mathrm{ml}$ d'inoculum et les logarithmes des nombres d'UFC/ml après une agitation standard des échantillons. Si $y$ est le nombre d'UFC/ml estimé à partir de la droite de calibrage, les limites de l'intervalle de confiance de $y$ (à $P=0,95)$ sont, en données non transformées : $y \cdot 10^{0,757}$ soit $y \cdot 5,71$ pour la limite supérieure et $y / 100,757$ soit $y / 5,71$ pour la limite inférieure.

D'autre part, les écarts types de répétabilité des temps de détection exprimés en $\log (\mathrm{UFC} / \mathrm{ml})$ varient peu en fonction de la dose d'inoculum; par contre, ils varient nettement en fonction du niveau de contamination des échantillons (tableau IV). L'écart type relatif des temps de détection (en h) est très faible $(2-4 \%)$ et constant à tous les niveaux de contamination; à l'inverse, après conversion en UFC/ml à partir

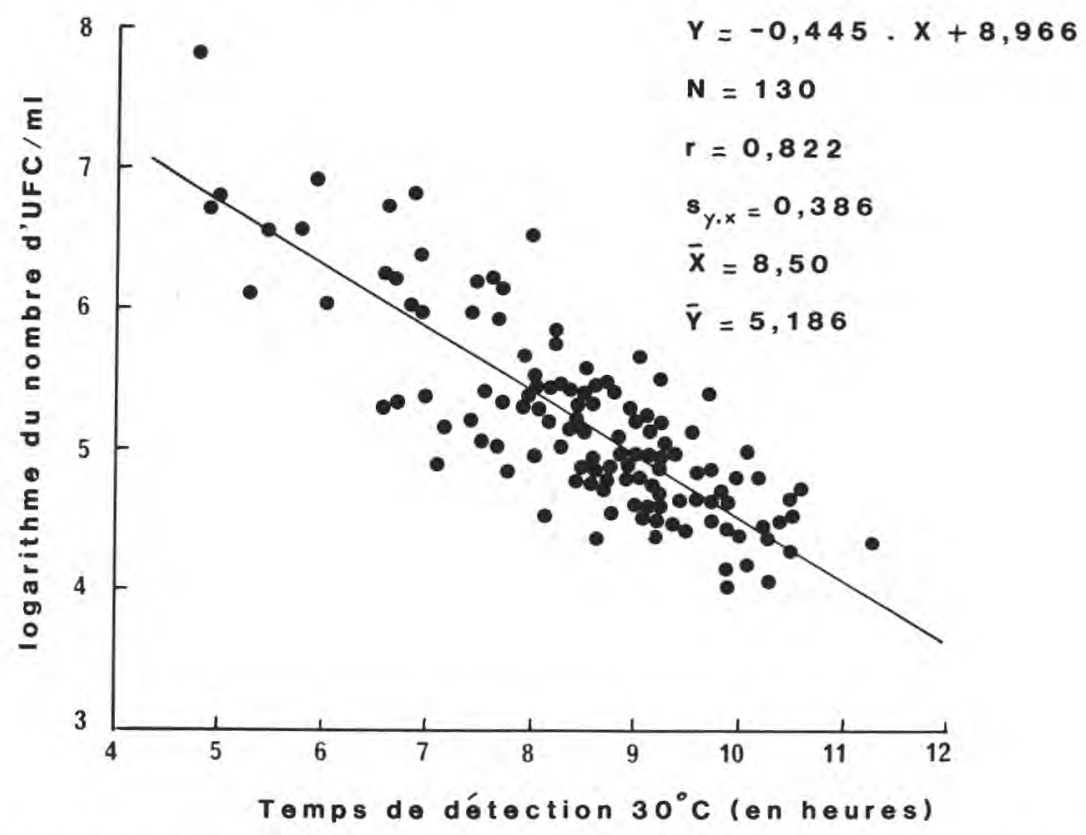

Fig 1. Relation entre les temps de détection obtenus à $30^{\circ} \mathrm{C}$ sur Spye Broth avec un inoculum de 0,1 $\mathrm{ml}$ et les logarithmes des nombres d'UFC/ml après une agitation standard des échantillons.

Relationship between the detection times measured at $30^{\circ} \mathrm{C}$ on Spye Broth with an inoculation volume of $0.1 \mathrm{ml}$ and the logarithms of the numbers of CFU/ml after "standard" shaking of samples. 
Tableau IV. Influence du niveau de contamination des échantillons de lait sur la répétabilité des temps de détection à $30^{\circ} \mathrm{C}$ sur milieu Spye Broth (Inoculum $0,1 \mathrm{ml}$ ).

Influence of the contamination level of the milk samples on the repeatability of the detection times obtained at $30^{\circ} \mathrm{C}$ on Spye Broth.

\begin{tabular}{llllrl}
\hline $\begin{array}{c}\text { Temps de détection } \\
\text { (h) }\end{array}$ & $\begin{array}{c}\text { Équivalence } \\
\left.\text { (UFC } \times 10^{3}\right)\end{array}$ & $\mathrm{N}$ & $\begin{array}{c}\mathrm{RSD} \\
\text { (h) }\end{array}$ & $\begin{array}{c}\mathrm{s}_{\mathrm{r}}{ }^{*} \\
\text { en } \log \text { (UFC) }\end{array}$ & $\begin{array}{c}\text { GRSD } \\
\text { (en UFC) }\end{array}$ \\
\hline Plus de 9,61 & Moins de 100 & 23 & $3,30 \%$ & 0,148 & $40,8 \%$ \\
$8,11-9,61$ & $100-500$ & 66 & $3,73 \%$ & 0,146 & $40,1 \%$ \\
$7,46-8,11$ & $500-1000$ & 16 & $2,60 \%$ & 0,084 & $21,3 \%$ \\
Moins de 7,46 & Plus de 1000 & 23 & $3,45 \%$ & 0,098 & $25,4 \%$ \\
& & & & & \\
Total & 128 & $3,50 \%$ & 0,133 & $35,9 \%$ \\
\hline
\end{tabular}

RSD : écart type relatif de répétabilité des temps de détection (en $\mathrm{h}$ ), repeatability relative standard deviation of the detection time (in h); $s_{r}^{*}$ : écart type de répétabilité des temps de détection (en log UFC/ml, calculé à partir de la pente de la droite de calibrage), repeatability standard deviation of the detection time in log (CFU/ml); GRSD: écart type relatif géométrique de répétabilité en UFC/ml, geometric relative standard deviation of repeatability in CFU/ml.

de la pente de la droite de calibrage, on note des écarts types relatifs géométriques de répétabilité élevés, surtout pour les faibles niveaux de contamination (jusqu'à plus de $40 \%$ ).

\section{Estimation des bactéries coliformes}

La figure 2 montre la relation obtenue, sur une population de 126 échantillons de lait cru, entre les temps de détection à $30^{\circ} \mathrm{C}$ sur Coliform Broth et les logarithmes des nombres de coliformes sur milieu VRBA après agitation du lait par turbine. L'équation de calibrage a été calculée après élimination de 4 échantillons qui s'écartaient de plus de 3 écarts types résiduels de la droite de calibrage. La précision d'estimation de la technique est donc de $\pm 1,96 \times$ 0,424 soit $\pm 0,831 \log$ (UFC/ml). Si $y$ est le nombre de coliformes estimé à partir de la droite de calibrage, les limites de l'inter- valle de confiance de $y$ (à $P=0,95)$ sont, en données non transformées :

$y / 6,78$ pour la limite inférieure

et $y \bullet 6,78$ pour la limite supérieure.

Conformément à ce qui a été observé pour la flore totale, l'écart type relatif de répétabilité des temps de détection (en $\mathrm{h}$ ) était faible (moins de $3 \%$ ) et restait constant, quel que soit le niveau de contamination du lait. Par contre, après conversion en UFC/ml, on notait une nette augmentation de l'écart type relatif géométrique quand le niveau de contamination du lait en coliformes baissait : en moyenne de $24,4 \%\left(s_{r}{ }^{*}=0,095 \log (\mathrm{UFC} / \mathrm{ml})\right]$, il atteignait $34,6 \%\left(s_{r}^{*}=0,129 \log (\mathrm{UFC} / \mathrm{ml})\right]$ audessous de $10^{3}$ coliformes $/ \mathrm{ml}$.

\section{Estimation de la flore psychrotrophe}

La figure 3 montre la relation obtenue entre les logarithmes des nombres de 


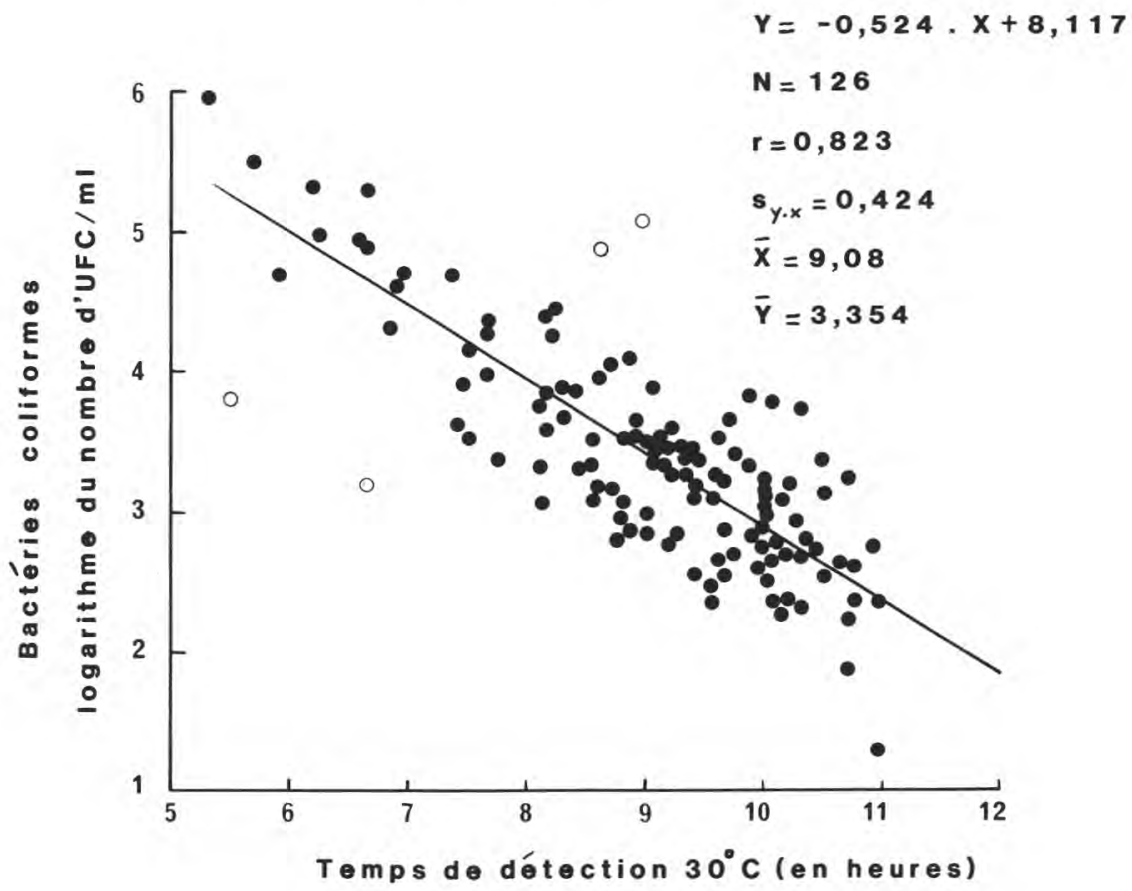

Fig 2. Relation entre les temps de détection mesurés à $30^{\circ} \mathrm{C}$ sur Coliform Broth et les logarithmes des nombres de coliformes obtenus sur milieu VRBA après une agitation par turbine des échantillons. La droite de calibrage a été calculée après élimination de 4 échantillons de lait (0) qui s'écartent de plus de 3 écarts types résiduels de cette droite.

Relationship between the detection times measured at $30^{\circ} \mathrm{C}$ on Coliform Broth and the logarithms of the numbers of CFU/ml obtained on Violet Red Bile Agar after shaking of samples with an Ultra-turrax.

micro-organismes psychrotrophes $/ \mathrm{ml}$ et les temps de détection, à $30^{\circ} \mathrm{C}$, sur milieu Spye Broth additionné de $2 \mathrm{mg} / \mathrm{l}$ de cristal violet. La précision d'estimation de la technique est assez peu satisfaisante : $\pm 1,96 \mathrm{x}$ 0,64 , soit $\pm 1,25 \log$ (UFC/ml). Ces temps de détection obtenus en présence de cristal violet ne permettaient pas d'estimer les niveaux de la flore à Gram négatif avec plus de précision : en effet, pour un inoculum de $0,1 \mathrm{ml}$, la relation obtenue entre ces temps et les logarithmes des nombres de micro-organismes à Gram négatif était la suivante :
$Y=-0,409 X+8,826$

avec $s_{y, x}=0,638$ et $r=0,662$.

Par contre, la figure 4 montre la relation obtenue, sur une population de 60 échantillons de lait, entre les logarithmes des nombres de micro-organismes psychrotrophes et les temps de détection mesurés à $10^{\circ} \mathrm{C}$ sur Spye Broth avec un inoculum de $1 \mathrm{ml}$ de lait. La relation entre les 2 techniques est relativement étroite puisque la précision d'estimation est de $\pm 1,96 \bullet 0,287$ soit $\pm 0,562 \log (U F C / m l)$. Si y est le niveau de la flore psychrotrophe estimé à partir de la droite de calibrage, les limites 


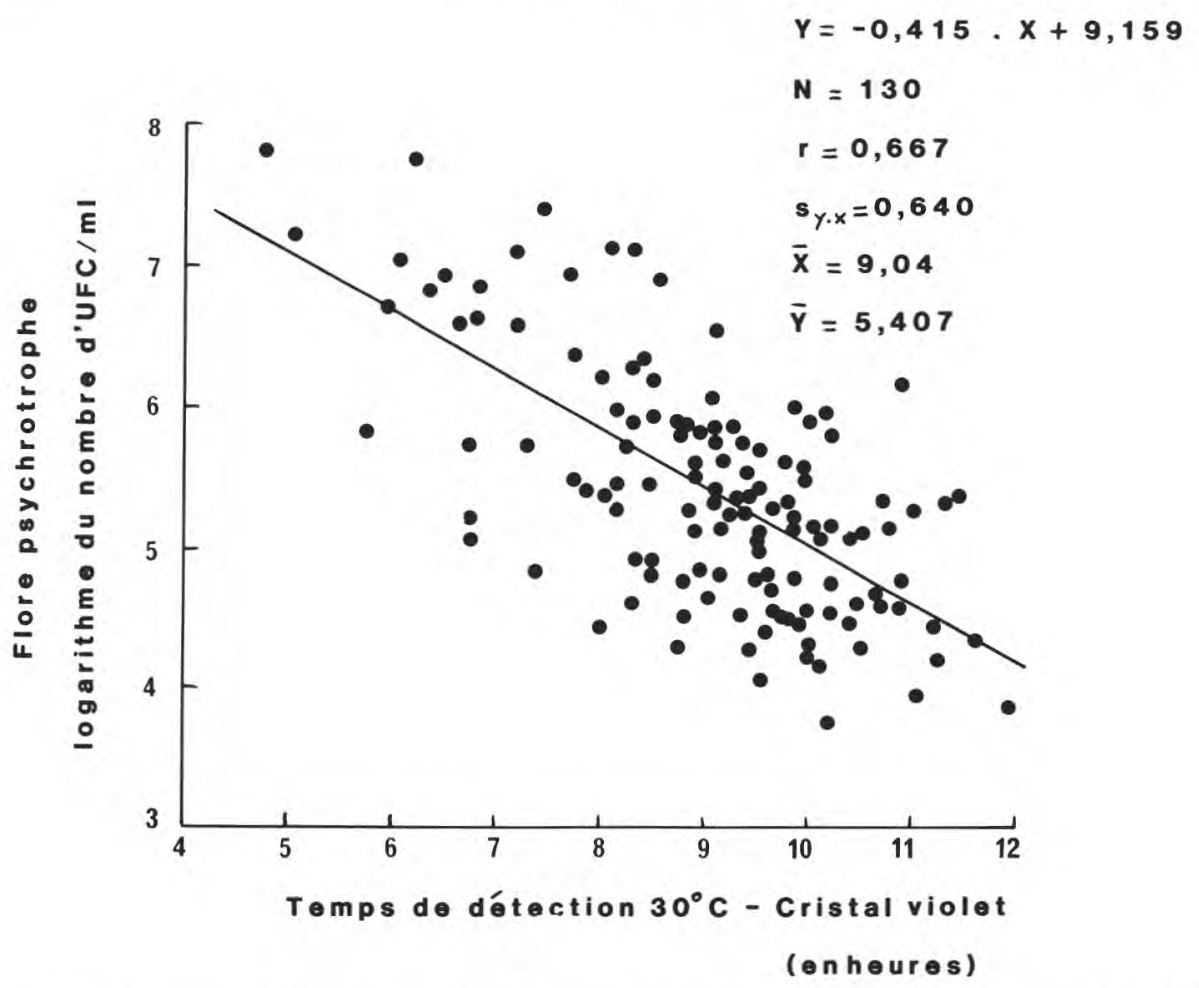

Fig 3. Relation entre les temps de détection obtenus à $30^{\circ} \mathrm{C}$ sur Spye Broth additionné de $2 \mathrm{mg} / \mathrm{lde}$ cristal violet (Inoculum de $0,1 \mathrm{ml}$ ) et les logarithmes des nombres de micro-organismes psychrotrophes après une agitation par turbine des échantillons.

Relationship between the detection times measured at $30{ }^{\circ} \mathrm{C}$ on Spye Broth supplemented with crystal violet (2 mg/l) and the logarithms of the numbers of psychrotrophic micro-organisms obtained after shaking of samples with an Ultra-turrax.

de l'intervalle de confiance de $y$ (à $P=$ $0,95)$ sont, en données non transformées : $y / 3,65$ pour la limite inférieure et $y \bullet 3,65$ pour la limite supérieure.

Contrairement à ce qui a été observé pour la flore totale, cette précision est légèrement meilleure avec un inoculum de $1 \mathrm{ml}$, bien que la différence ne soit pas significative (test $F$ de comparaison des variances à $n-2$ ddl; Dagnelie, 1970).

Enfin, la répétabilité des temps de détection à $10^{\circ} \mathrm{C}$ était très proche de celle observée pour les 2 groupes microbiens précédents : pour des inoculums de 1 et $0,1 \mathrm{ml}$, les écarts types de répétabilité $s_{r}^{*}$ étaient respectivement de 0,128 et de $0,121 \log (\mathrm{UFC} / \mathrm{ml})$ ce qui correspond à des écarts types relatifs géométriques de 34,2 et $32,2 \%$.

\section{DISCUSSION ET CONCLUSION}

Avec un écart type résiduel de 0,39 log (UFC/ml), la justesse de l'appareil Malthus 


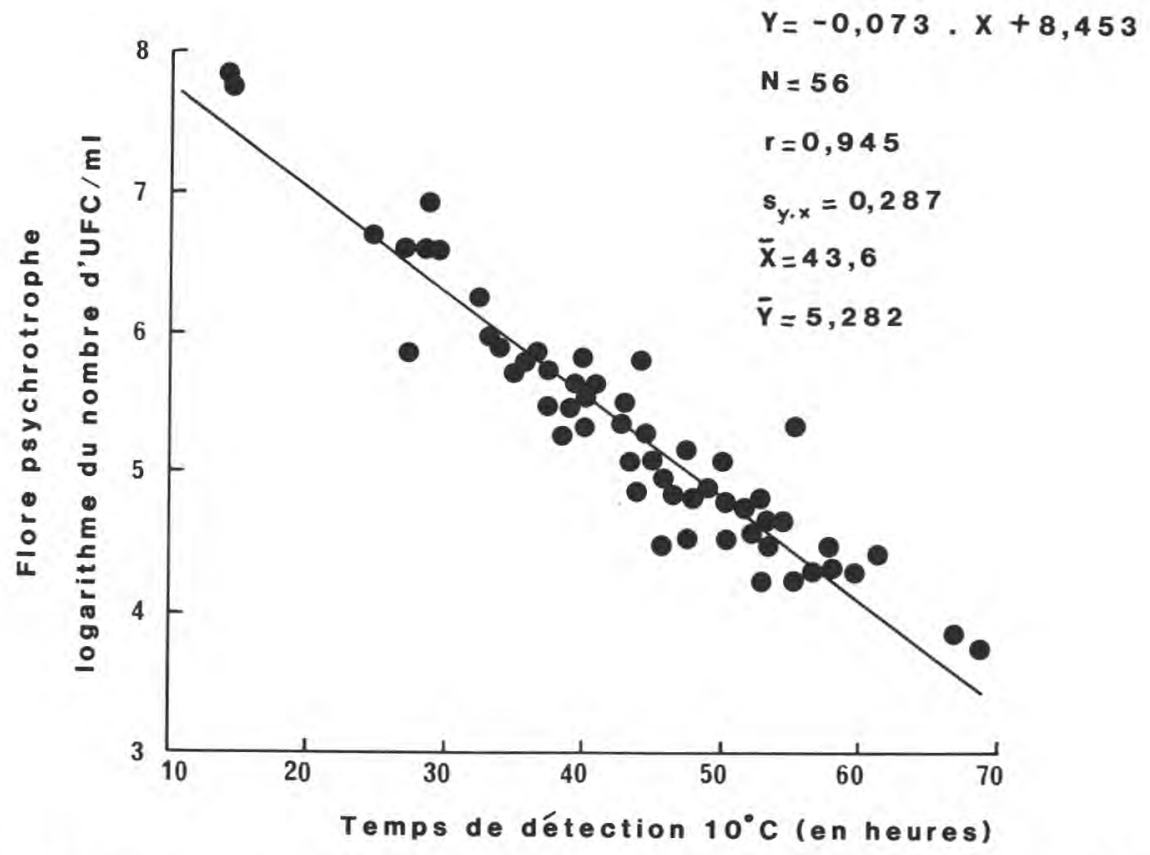

Fig 4. Relation entre les temps de détection obtenus à $10^{\circ} \mathrm{C}$ sur milieu Spye Broth (Inoculum $1 \mathrm{ml}$ ) et les logarithmes des nombres de micro-organismes psychrotrophes après une agitation par turbine des échantillons.

Relationship between the detection times measured at $10^{\circ} \mathrm{C}$ on Spye Broth (Inoculation volume of $1 \mathrm{ml}$ ) and the logarithms of the numbers of psychrotrophic micro-organisms obtained after shaking of samples with an Ultra-turrax.

pour l'estimation de la flore totale du lait cru est équivalente à celle obtenue dans les études de Nieuwenhof et Hoolwerf (1987) et Piton et Dasen (1988) sur le Bactometer 123 (tableau I). En effet, si les résultats semblent légèrement meilleurs pour le Malthus, il faut souligner que l'écart type résiduel peut varier dans des proportions assez larges entre essais effectués dans les mêmes conditions expérimentales sur 2 populations différentes d'échantillons de lait. Ainsi Piton et Dasen (1988) ont obtenu un écart type résiduel significativement plus faible dans le cas d'échantillons prélevés dans des citernes de ramassage d'entreprises $[0,37 \log (\mathrm{UFC} / \mathrm{ml})]$ que dans le cas d'échantillons prélevés dans des tanks de ferme $[0,47 \log (U F C / m l)] ;$ de même, entre 2 populations différentes d'échantillons prélevés à la ferme, ils ont observé des variations de cet écart type atteignant près de $0,10 \log (\mathrm{UFC} / \mathrm{ml})$. Des variations dans la nature et/ou l'état physiologique de la flore bactérienne du lait sont probablement à l'origine de ces différences.

Un mode d'agitation violent des échantillons avant la numération microbienne entraîne une dégradation de la précision d'estimation des mesures de conductance; d'autre part, d'après Nieuwenhof et Hoolwerf (1987) et Piton et Dasen (1988), on observe des précisions équivalentes si les analyses microbiologiques et les mesures de conductance sont effectuées 
avec le même mode d'agitation des échantillons (standard ou turbine). Ces 2 observations sont contraires à ce qu'on pouvait attendre, puisque le mode d'agitation par Ultra-turrax permet une désagrégation maximale des amas microbiens et donc une meilleure estimation des nombres de micro-organismes présents (Richard, 1980). Les résultats obtenus par conductimétrie semblent donc davantage reliés au nombre d'amas microbiens qu'au nombre réel de micro-organismes. Cela peut sans doute être attribué au fait que le changement de conductance du milieu est à la fois fonction du nombre initial de microorganismes et de leur activité dans le milieu de culture (Firstenberg-Eden et Eden, 1984; Suhren et Heeschen, 1985). Ainsi, il a été montré (Hadley et Senyk, 1975; Firstenberg-Eden et Eden, 1984; Suhren et Heeschen, 1985) que le nombre de bactéries atteint dans le milieu de culture au moment de ce changement varie dans des proportions assez larges (entre $10^{6}$ et $10^{8}$ cellules $/ \mathrm{ml}$ ); en revanche, d'après Suhren et Heeschen (1985), ce changement semble lié à une nette augmentation de la concentration en ammoniac du milieu. II est donc probable que les cellules emprisonnées dans les amas microbiens aient une activité moindre sur les substrats du milieu que des cellules isolées. D'autre part, il est possible que ces cellules emprisonnées se multiplient moins vite dans le milieu, en raison de conditions de croissance défavorables (disponibilité en nutriments et en oxygène).

Ces observations sur l'influence du mode d'agitation permettent alors de conclure que la justesse obtenue au cours de notre essai pour l'estimation des bactéries coliformes ou de la flore psychrotrophe est en réalité nettement sousestimée; en effet, les dénombrements de ces 2 flores ont été effectués après une agitation par turbine des échantillons. Or, Piton et Richard (1983) ont montré que pour les Pseudomonas psychrotrophes et les bactéries coliformes, une agitation par turbine pouvait conduire à une multiplication très importante des nombres d'UFC/ml par rapport à un mode d'agitation peu énergique (souvent 20 et parfois près de 100 fois plus).

Pour l'estimation des bactéries coliformes, les mesures de conductance à $30{ }^{\circ} \mathrm{C}$ présentent une précision d'estimation équivalente à celle que l'on peut observer pour la flore totale $\left[s_{y, x}=0,424 \mathrm{log}\right.$ (UFC/ml)]. Plusieurs hypothèses peuvent expliquer ce manque d'étroitesse entre les 2 techniques : tout d'abord, les variations de nature de la flore coliforme d'un échantillon à l'autre; ensuite, le mode d'agitation des échantillons avant leur numération et enfin, la croissance de bactéries non coliformes dans le milieu. En particulier, on sait que des micro-organismes à Gram négatif lactose moins (Pseudomonas psychrotrophes, en particulier) sont capables de croître sur les milieux de dénombrement des coliformes (Richard et al, 1988). II est donc vraisemblable, qu'à la température choisie pour l'incubation des cellules $\left(30^{\circ} \mathrm{C}\right)$, les conditions d'anaérobiose partielle rencontrées dans les cellules de $10 \mathrm{ml}$ et les constituants du milieu de culture n'aient pas permis une inhibition totale de ces micro-organismes. L'augmentation de la température d'incubation aurait permis d'empêcher la croissance de la plupart de ces micro-organismes : à $35^{\circ} \mathrm{C}$ par exemple, comme dans l'étude de Firstenberg-Eden et al (1984). Mais à l'inverse, cette élévation de la température d'incubation aurait probablement inhibé la croissance des bactéries coliformes psychrotrophes.

Par ailleurs, grâce à un abaissement de la température d'incubation des cellules à $10^{\circ} \mathrm{C}$, la conductimétrie permet d'évaluer le niveau de la flore psychrotrophe avec une précision d'estimation relativement satisfaisante $\left[s_{y, x}=0,287 \log (\mathrm{UFC} / \mathrm{ml})\right]$. 
Cette technique présente néanmoins l'inconvénient de temps de détection très longs (en moyenne, près de $48 \mathrm{~h}$ ). Cette relation assez étroite peut être expliquée principalement par 2 faits : d'une part, l'inhibition de la croissance des bactéries mésophiles; d'autre part, l'homogénéité de la flore psychrotrophe des échantillons de lait, sur le plan de sa nature et de son état physiologique, puisque la conservation du lait pendant des durées assez longues à basse température entraîne la prolifération d'un seul groupe de micro-organismes, les Pseudomonas psychrotrophes (Richard, 1981). L'utilisation d'un inhibiteur de la flore à Gram positif à $30^{\circ} \mathrm{C}$ n'a, par contre, pas permis d'estimer avec précision les niveaux de la flore psychrotrophe. Une température d'incubation plus basse (18 ou $20^{\circ} \mathrm{C}$ ) aurait sans doute été préférable; d'autre part, le mode d'agitation violent des échantillons avant leur numération a pu jouer un grand rôle en défaveur de la précision de cette technique, puisque les bactéries psychrotrophes se présentent assez souvent sous forme d'amas très faciles à dissocier.

Enfin, la répétabilité des temps de détection du Malthus varie peu en fonction du groupe microbien recherché et des conditions expérimentales. Dans le cas de la flore totale, l'écart type de répétabilité en $\log$ (UFC) est d'environ 0,130 , ce qui correspond à un écart type relatif géométrique de $35 \%$. Cette répétabilité est donc nettement moins satisfaisante que celle des techniques de référence puisque la norme généralement retenue pour ces techniques est de $0,07 \mathrm{log}$ (UFC/ml) (Anonyme, 1978). Par contre, ces caractéristiques de répétabilité sont très proches de celles observées sur le Bactometer avec la technique d'ensemencement en surface (OnMPCA sur le tableau I) (Nieuwenhof et Hoolwerf, 1987; Piton et Dasen, 1988). Cependant, ces auteurs ont pu nettement améliorer l'écart type de répétabilité des temps de détection avec la technique d'ensemencement en profondeur (In-MPCA) : moins de $0,07 \log$ (UFC) à tous les niveaux de contamination du lait. Enfin, pour l'estimation des bactéries coliformes, les écarts types de répétabilité sont, en revanche, meilleurs que ceux généralement obtenus avec les techniques de référence : $0,146 \log$ (UFC/ml) d'après Ginn et al (1986) ou $0,219 \mathrm{log}$ (UFC/ml) d'après Piton et Grappin (1990).

\section{REMERCIEMENTS}

Ce travail a reçu le soutien financier de la Direction générale de l'alimentation du ministère de l'Agriculture (Contrat DGAL $n^{\circ}$ R88/11).

\section{RÉFÉRENCES}

Anonyme (1978) Standard Methods for the Examination of Dairy Products. American Public Health Association, 14th edn, New York

Anonyme (1985) Milk. Definition and Evaluation of the Overall Accuracy of Indirect Methods of milk analysis. Application to Calibration Procedure and Quality Control in Dairy Laboratory. Norme provisoire FIL-IDF 128

Cady P, Hardy D, Martins S, Dufour SW, Kraeger SJ (1978) Automated impedance measurements for rapid screening of milk microbial content. J Food Prot 41, 277-283

Campbell JE, Gilchrist JE (1973) Spiral plate technique for counting bacteria in milk and other foods. Dev Ind Microbiol 14, 95-102

Coombs JP (1989) An electrical method to detect psychrotrophic bacteria in raw milk. In: Rapid Methods and Automation in Microbiology and Immunology. 5th int symposium, Florence, 1987 (Balows A, Tilton RC, Turano A, eds) Brixia Academic Press, Brescia, 624627

Dagnelie P (1970) Théorie et Méthodes Statistiques. Vol 2. Les Méthodes de l'inférence statistique. Duculot SA, Gembloux 
Donnelly CB, Gilchrist JE, Peeler JT, Campbell JE (1976) Spiral plate count method for the examination of raw and pasteurized milk. Appl Environ Microbiol 32, 21-27

Firstenberg-Eden R (1984) Collaborative study of the impedance method for examining raw milk samples. J Food Prot 47, 407-412

Firstenberg-Eden R, Tricarico M (1983) Impedimetric determination of total, mesophilic and psychrotrophic counts in raw milk. J Food Sci $48,1750-1754$

Firstenberg-Eden R, Eden G (1984) Impedance Microbiology. Research Studies Press Ltd, John Wiley \& Sons Inc, Chichester

Firstenberg-Eden R, Zindulis J (1984) Electrochemical changes in media due to microbial growth. J Microbiol Methods 2, 103-115

Firstenberg-Eden R, Van Sise ML, Zindulis $\mathrm{J}$, Kahn P (1984) Impedimetric estimation of coliforms in dairy products. I Food Sci 49, 1449-1452

Gilchrist JE, Campbell JE, Donnelly CB, Peeler JT, Delaney JM (1973) Spiral plate method for bacterial determination. Appl Microbiol $25,244-252$

Ginn RE, Packard VS, Fox TL (1986) Enumeration of total bacteria and coliforms in milk by rehydratable film methods: collaborative study. J Assoc Off Anal Chem 69, 527-531

Gnan S, Luedecke LO (1982) Impedance measurements in raw milk as an alternative to the standard plate count. J Food Prot 45, 4-7

Hadley WK, Senyk G (1975) Early detection of microbial metabolism and growth by measurement of electrical impedance. In: Microbiology. Am Soc Microbiol, Washington, DC, 12-21

Jarvis B, Lach VH, Wood JM (1977) Evaluation of the spiral plate maker for the enumeration of microorganisms in foods. $J$ Appl Microbiol 43, 149-157

Nieuwenhof FFJ, Hoolwerf JD (1987) Impedance measurement as an alternative to the plate count method for estimating the total count of bacteria in raw milk. $J$ Food Prot 50 , 665-668
O'Connor F (1979) An impedance method for the determination of bacteriological quality of raw milk. Ir J Food Sci Technol 3, 93-100

Peeler JT, Gilchrist JE, Donnelly CB, Campbell JE (1977) A collaborative study of the spiral plate method for examining milk samples. $J$ Food Prot 40, 462-464

Piton C, Richard J (1983) Influence de l'agitation des échantillons de lait cru sur les résultats de dénombrement de 3 groupes microbiens d'intérêt technologique. Lait 63, 405-415

Piton C, Dasen A (1988) Evaluation de la mesure d'impédance comme technique rapide d'appréciation de la qualité bactériologique du lait cru. Lait 68, 467-484

Piton C, Grappin R (1990) A model for statistical evaluation of precision parameters of microbiological methods: application to the dry rehydratable film methods and the IDF reference methods for the enumeration of total aerobic mesophilic flora and coliforms in raw milk. J Assoc Off Anal Chem (submitted)

Richard J (1980) Influence de l'agitation du lait cru sur les résultats de dénombrement de sa flore totale à l'aide d'une anse calibrée. Lait $60,211-225$

Richard J (1981) Influence de diverses méthodes de nettoyage des machines à traire sur la "qualité de conservation" du lait cru à basse température. Lait 61, 354-369

Richard J, Krebs C, Walter J, Grappin R (1981) Comparaison de divers modes d'agitation des échantillons de lait cru en vue de la numération de sa flore totale suivant la méthode Thompson. Lait 61, 250-260

Richard J, Piton C, Gratadoux JJ (1988) Nature des colonies se développant à la surface du milieu Violet Red Bile Lactose Agar (VRB) ensemencé avec du lait cru. Validité des dénombrements des colonies lactose-positives et des colonies lactose-négatives se développant sur ce milieu. Lait 68, 311-322

Suhren G, Heeschen W (1985) Zur Impedanz als Messgrösse für die bakteriologische Untersuchung von Milch und Milchprodukten. Dtsch Molk Ztg 51/52, 1710-1717 